\title{
FRAGMENTATION OF MOLECULAR CLOUDS AND STAR FORMATION
}

\author{
E. FALGARONE, F. BOULANGER AND G. DUVERT (EDS.)
}

The structure of the cold interstellar medium and its impact on star formation is emerging as a new field of investigation. This is largely due to the recent advent of telescopes sensitive in the millimeter, submillimeter and infrared ranges. Small scale structure information is now accessible, not only on the most conspicuously active sites of star formation, but also on extended regions of the placental material. This allows astronomers to adopt a global approach to the physics of star formation.

This book records all the contributions to IAU Symposium No. 147, which presented studies of the multi-scale processes at work in fragmentation of interstellar clouds, and the triggering of star formation.

The topics range from large-scale to small-scale structures in which several physical and chemical processes occur. Star formation and protostar evolution are then covered as a result. 